\title{
MUTAMICINS; BIOSYNTHETICALLY CREATED NEW SISOMICIN ANALOGUES
}

\author{
R.T. Testa, G.H. Wagman, P.J.L. Daniels and M.J. Weinstein \\ Microbiological and Medicinal Chemistry Divisions, Schering Corporation, \\ Bloomfield, N. J., 07003 U.S.A.
}

(Received for publication September 17, 1974)

\begin{abstract}
A mutant of Micromonospora inyoensis, the sisomicin-producing organism, has been isolated which requires the addition of 2-deoxystreptamine to the fermentation broth for sisomicin production. The addition of analogues of 2-deoxystreptamine to this mutant resulted in the formation of new antibiotics called mutamicins. Mutamicin 1 , produced by the addition of streptamine to the fermentation broth, exhibits broad-spectrum activity with potency similar to or slightly less than sisomicin, with the exception of gentamicin-sisomicin-adenylylating strains against which mutamicin 1 exhibited greatly improved activity. Mutamicin 2, produced by the addition of 2, 5-dideoxystreptamine, exhibits similar broad-spectrum activity but with favorable activity against gentamicin-sisomicin-acetylating strains.
\end{abstract}

Sisomicin belongs to the deoxystreptamine-containing aminoglycoside group of antibiotics ${ }^{1)}$. Using Streptomyces fradiae, a neomycin-producer, SHIER et al. ${ }^{2)}$ developed a novel method for producing semisynthetic aminoglycoside antibiotics by isolating mutants which require 2-deoxystreptamine for antibiotic production. Streptamine and 2-epistreptamine, analogues of 2-deoxystreptamine, were shown to be converted by this mutant into analogues of neomycin, termed hybrimycins. This group also demonstrated the same phenomenon with the paromomycinproducer, S. rimosus forma paromomycinus, and to some extent with the kanamycin-producer S. kanamyceticus $^{3)}$. A similar response has recently been shown by KoJImA and SAToH ${ }^{4)}$ with deoxystreptamine-requiring mutants of the ribostamycin-producer, S. ribosidificus, and the kanamycin-producer, S. kanamyceticus; by CLARIDGE et al. ${ }^{5)}$ with the butirosin-producer, Bacillus circulans; and by NAGAOKA and Demain ${ }^{6)}$ with the streptomycin-producer, S. griseus.

This report describes the isolation of a 2-deoxystreptamine-requiring mutant from Micromonospora inyoensis, the sisomicin producer, and the conversion of various 2-deoxystreptamine analogues into new sisomicin analogues.

\section{Materials and Methods}

Organism and Culture Conditions

Micromonospora inyoensis (NRRL 3292) was grown on an agar medium consisting of (per liter): yeast extract, $5 \mathrm{~g}$; corn steep liquor solids, $1 \mathrm{~g}$; fish solubles, $1 \mathrm{~g}$; and calcium carbonate $4 \mathrm{~g}$, for approximately 2 weeks at $28^{\circ} \mathrm{C}$ for the preparation of spores. The spores were harvested and used for all mutation studies.

Inocula for fermentations were prepared in a medium with the following composition (per liter): yeast extract, $5 \mathrm{~g}$; beef extract, $3 \mathrm{~g}$; tryptose, $5 \mathrm{~g}$; starch, $24 \mathrm{~g}$; dextrose, $5 \mathrm{~g}$; and calcium carbonate, $4 \mathrm{~g}$. The culture is grown for 3 days at $35^{\circ} \mathrm{C}$ on a rotary shaker at approximately $300 \mathrm{rpm}$. Fermentations were carried out at $28^{\circ} \mathrm{C}$ for $5 \sim 7$ days on a rotary shaker in a medium with the following composition (per liter): soybean grits, $35 \mathrm{~g}$; dextrin, $50 \mathrm{~g}$; dextrose $5 \mathrm{~g}$; calcium carbonate, $7 \mathrm{~g}$; and cobalt chloride $24 \mathrm{mg}$. 
Mutation Procedures

Clean spore preparations were used for exposure to various mutagens including $\mathrm{N}$-methyl$\mathrm{N}^{\prime}$-nitro-N-nitroso-guanidine, ethylmethane sulfonate, and ultraviolet light.

The mutant was selected using a procedure similar to that described by SHIER et al. ${ }^{2)}$ The survivors were replicated onto an agar medium with and without $400 \mu \mathrm{g} / \mathrm{ml}$ of 2-deoxystreptamine added. The medium consisted of (per liter): yeast extract, $5 \mathrm{~g}$; meat extract, $3 \mathrm{~g}$; corn steep liquor solids, $1 \mathrm{~g}$; soluble starch, $20 \mathrm{~g}$; calcium carbonate, $4 \mathrm{~g}$; and agar, $15 \mathrm{~g}$. The agar plates were incubated for $3 \sim 5$ days, after which time they were overlaid with agar seeded with Staphylococcus aureus and incubated overnight at $37^{\circ} \mathrm{C}$. The colony showing a zone of inhibition on the test plate (2-deoxystreptamine added) but no zone on the control plate (no 2-deoxystreptamine added) was selected as a mutant requiring the addition of 2-deoxystreptamine for antibiotic production. The requirement was checked by flask fermentation as described.

\section{Detection of Mutamicins}

Preliminary detection of antibiotic activity was determined by disc testing of broth extracts against $S$. aureus. Paper chromatography in a solvent system consisting of chloroform-methanol $-17 \%$ ammonium hydroxide $(2: 1: 1, \mathrm{v} / \mathrm{v})$ followed by bioautography was used for preliminary determination of a new antibiotic. The lower solvent phase was used.

Absolute structural determinations of the mutamicins was carried by NMR and mass spectroscopy and will be the subject of a separate report (DANIELS and YeHASKEL, paper in preparation).

Isolation of the Mutamicins

The antibiotics were removed from acidified fermentation broths by an ion-exchange procedure using Amberlite IRC-50 resin according to the method described for sisomicin ${ }^{7}$. The resin eluates containing the antibiotic were evaporated to dryness and this was used for biological testing.

\section{In vitro Testing}

The antibiotics prepared as described above were tested by conventional tube dilution tests in Mueller-Hinton broth.

\section{Results and Discussion}

A deoxystreptamine-requiring mutant of $M$. inyoensis (1550F) was isolated which produced antibiotic only when 2-deoxystreptamine was added to the culture medium. The antibiotic produced in this manner was isolated and shown to be sisomicin by comparative chromatography, IR and NMR spectroscopy with an authentic sisomicin sample.

The addition of analogues of deoxystreptamine to the culture medium, however, resulted in the formation of new sisomicin analogues designated as mutamicins. A list of the compounds tested and their structures is given in Fig. 1.

As noted with the neomycin, paromomycin, and the ribostamycin cultures ${ }^{2,3,4)}$, the addition of streptamine (A) resulted in the formation of a new antibiotic (Figs. 2 and 3). This antibiotic, termed mutamicin 1, was isolated and tested against a number of sensitive and resistant bacterial cultures, by tube dilution (Table 1). The MIC's show similar or slightly less activity compared to sisomicin against both gram-positive and gram-negative microorganisms including kanamycin-phosphorylating Klebsiella strains, and a tobramycin-resistant gentamicin-sensitive E. coli. However, mutamicin 1 is active against $E$. coli and $K$. pneumoniae strains containing the gentamicin, tobramycin, sisomicin adenylylating $\mathrm{R}$-factor but not against the gentamicin-acetylating Pseudomonas strains. 
Fig. 1. Compounds tested for conversion into mutamicins

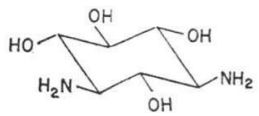

A. Streptamine

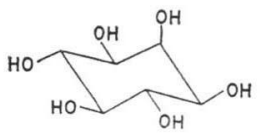

C. Myoinositol

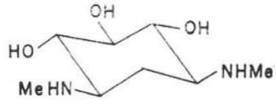

E. 1,3-Di-N-methyl-2-deoxystreptomine

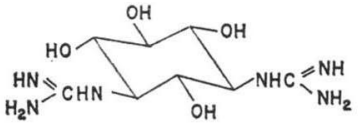

G. Streptidine

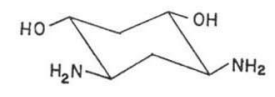

B. 2.5-Dideoxystreptamine

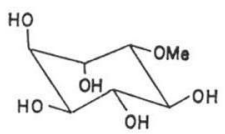

D. Quebrachitol

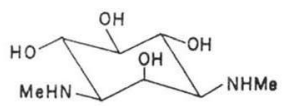

F. Actinamine

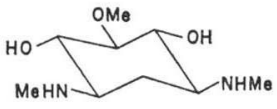

H. 1.3.5 - Tri - N, N,O-methyl2 -deoxystreptamine

Fig. 3. Structures of mutamicins 1 and 2

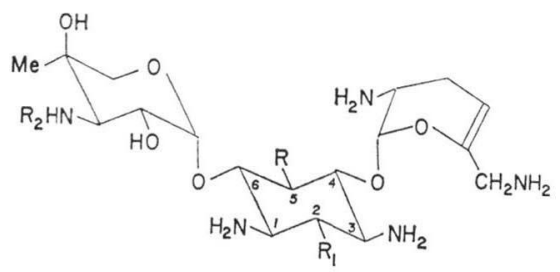

$$
\begin{array}{ll}
\text { Sisomicin } & R=O H, \quad R_{1}=H, R_{2}=M e \\
\text { Mutomicin I } & R=R_{1}=O H, R_{2}=M e \\
\text { Mutamicin Io } & R=R_{1}=O H, \quad R_{2}=C O M e \\
\text { Mutamicin Ib } & R=R_{1}=O H, R_{2}=H \\
\text { Mutamicin 2 } & R=R_{1}=H, \quad R_{2}=M e
\end{array}
$$

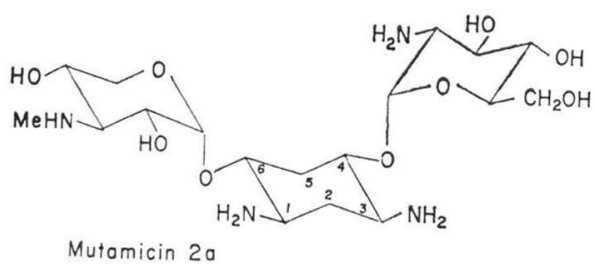

Fig. 2. Bioautograph of mutamicins compared to sisomicin. Solvent system: ChloroformMethanol- $17 \%$ ammonium hydroxide $(2: 1: 1)$

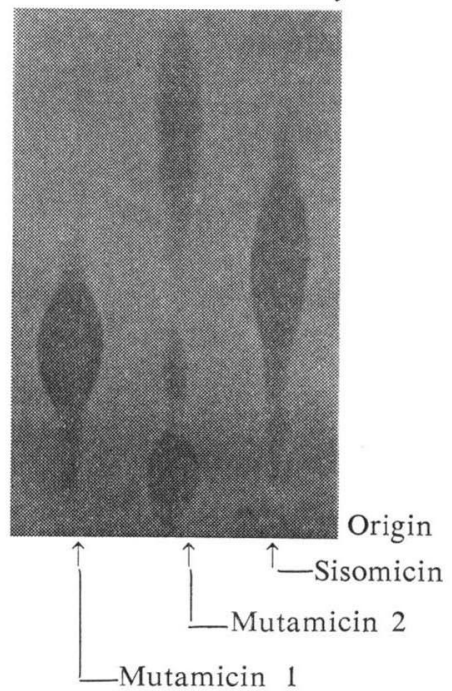

A heretofore undescribed aminocyclitol, 2,5-dideoxystreptamine (B), produced by a novel method (paper in preparation), was incorporated and converted into novel antibiotics termed mutamicin 2 and $2 \mathrm{a}$ (Fig. 2), the structures of which are shown in Fig. 3. This is the first instance demonstrating a change in the 5 position of 2-deoxystreptamine which leads to an active antibiotic. As can be also seen in Table 1 , mutamicin 2 exhibits activity similar to sisomicin but significantly, it is active against the gentamicinsisomicin-acetylating strains. However, it is not active against the adenylylating $\mathrm{R}$-factor strains. From preliminary results, based upon disc testing only using the BAUER-KIRBY technique, the mutamicin 2 complex exhibited

activity against the gentamicin-resistant E. coli strains JR88 and 90 . These strains have been found to acetylate gentamicin at N-3 of the 2-deoxystreptamine part of the molecule. No results are yet available on the activity of mutamicins $1 \mathrm{a}, 1 \mathrm{~b}$ and $2 \mathrm{a}$.

Several analogues were tested which did not result in the formation of a biologically-active compound $(\mathrm{C}-\mathrm{H})$. As can be seen, myo-inositol $(\mathrm{C})$, which has been reported as a precursor to streptamine ${ }^{\theta, 10)}$ was not incorporated into an active compound. Analogues of 2-deoxystreptamine in which the both of the $\mathrm{NH}_{2}$ group are methylated $(\mathrm{E}, \mathrm{F}, \mathrm{H})$ also do not form antibiotics as was recently shown by SHIER et al. ${ }^{8)}$ with the neomycin, paromomycin, and kana- 
Table 1. In vitro Activity of mutamicin 1,2 and sisomicin MIC's in Mueller-Hinton broth $\mathrm{pH} 7.2$

\begin{tabular}{|c|c|c|c|c|}
\hline \multirow{2}{*}{\multicolumn{2}{|c|}{ Organism }} & \multicolumn{3}{|c|}{$\mathrm{MIC}(\mathrm{mcg} / \mathrm{ml})$} \\
\hline & & Mutamicin 1 & Mutamicin 2 & Sisomicin \\
\hline \multirow[t]{4}{*}{ Staphylococcus aureus } & $209 \mathrm{P}$ & 0.3 & 0.08 & 0.08 \\
\hline & Wood & 0.08 & 0.08 & 0.03 \\
\hline & Ziegler & 0.08 & 0.3 & 0.03 \\
\hline & $59 \mathrm{~N}$ & 0.3 & 0.3 & 0.03 \\
\hline \multirow[t]{4}{*}{ Streptococcus pyogenes } & $\mathrm{C}$ & 3.0 & 17.5 & 3.0 \\
\hline & 27 & 7.5 & 17.5 & 3.0 \\
\hline & Cruz & 3.0 & 7.5 & 3.0 \\
\hline & Alvarez & 7.5 & 7.5 & 3.0 \\
\hline Escherichia coli & 10536 & 0.3 & 0.3 & 0.08 \\
\hline G/S/T-Res. (adenyl.) & W677/R55 & 0.8 & 17.5 & 7.5 \\
\hline " $\quad "$ & LA290/R 55 & 3.0 & 17.5 & 17.5 \\
\hline \multirow[t]{4}{*}{ K-Res. (phos.) } & 589 & 3.0 & 3.0 & 0.8 \\
\hline & C-13 & 3.0 & 0.75 & 0.3 \\
\hline & Baker 2 & 3.0 & 0.75 & 0.3 \\
\hline & F14-Bk & 3.0 & 3.0 & 0.3 \\
\hline T-Res. & 4195 & 3.0 & 17.5 & 0.8 \\
\hline Klebsiella pneumoniae & AD13 & 0.3 & 0.3 & 0.08 \\
\hline K-Res. (phos.) & AD22 & 0.3 & 0.8 & 0.3 \\
\hline \multirow[t]{3}{*}{ G/S/T-Res. (adenyl.) } & 3694 & 0.3 & 17.5 & 7.5 \\
\hline & 3020 & 0.3 & 17.5 & 7.5 \\
\hline & 121 & 3.0 & 25 & 0.08 \\
\hline \multirow[t]{5}{*}{ Pseudomonas aeruginosa } & 1262 & 3.0 & 3.0 & 0.8 \\
\hline & 762 & 3.0 & 0.8 & 0.3 \\
\hline & 1395 & 3.0 & 0.3 & 0.08 \\
\hline & 3223 & 0.3 & 0.3 & 0.08 \\
\hline & D-2 & 0.8 & 3.0 & 0.08 \\
\hline \multicolumn{2}{|l|}{ G/S/T-Res. (Travers) } & $>25$ & $>25$ & $>25$ \\
\hline G/S/Res. (acetyl.) & Stone 130 & 17.5 & 3.0 & 17.5 \\
\hline \multirow[t]{2}{*}{$" \prime$} & 138 & 17.5 & 3.0 & 7.5 \\
\hline & 20 & 3.0 & 0.3 & 0.3 \\
\hline$"$ & Capetown 18 & 7.5 & 0.8 & 7.5 \\
\hline Proteus mirabilis & & 3.0 & 3.0 & 0.3 \\
\hline Proteus rettgeri & & 3.0 & 0.8 & 0.8 \\
\hline Providence 164 (G/S-Res) & & $>25$ & $>25$ & $>25$ \\
\hline Salmonella typhimurium & & 3.0 & 0.8 & 0.3 \\
\hline Serratia marcescens & 127 & 0.8 & 3.0 & 0.8 \\
\hline
\end{tabular}

$\mathrm{G}=$ gentamicin; $\mathrm{K}=$ kanamycin; $\mathrm{S}=$ sisomicin; $\mathrm{T}=$ tobramycin; Res=resistant $\operatorname{strain}$

mycin mutants.

These results indicate a valuable approach to the formation of antibiotics with desirable antibacterial activity. Testing is continuing for the production of additional novel antibiotics using this technique. From a study of the compounds which were found to be incorporated and the activity of the antibiotics produced, as well as those analogues which are not incor- 
porated, it is possible to gain more insight as to structure-activity relationships as well as methods whereby certain toxic effects may be altered.

\section{Acknowledgements}

We gratefully acknowledge the capable assistance of Ms. Lois Kamnitzer, Mr. Eugene Moss, and Mr. A YeHASKel.

\section{References}

1) Weinstein, M.J.; J.A. Marquez, R.T. Testa, G.H. Wagman, E.M. Oden \& J.A. Waitz: Antibiotic 6640, a new Micromonospora-produced aminoglycoside antibiotic. J. Antibiotics 23: 551 $\sim 554,1970$

2) Shier, W. T.; K. L. Rinehart, Jr. \& D. Gottlieb: Preparation of four new antibiotics from a mutant of Streptomyces fradiae. Nat. Acad. Sci. 63: 198 204, 1969

3) Shier, W. T.; K. L. Rinehart, Jr. \& D. Gottlieb: Method of making antibiotics containing the aminocyclitol subunit. U.S. Patent 3,669,838, June 13, 1972; CA 77: 150584x. 1972

4) KoJima, M. \& A. Satoh: Microbial biosynthesis of aminoglycosidic antibiotics by mutants of S. ribosidificus and S. kanamyceticus. J. Antibiotics 26: 784 786, 1973

5) Claridge, C.A.; J.A. Bush, M.D. Defuria \& K.E. Price: Fermentation and mutation studies with a butirosin-producing strain of Bacillus circulans. Devel. in Industrial Microbiol. 15: 101 113,1974

6) Nagaoka, K. \& A. L. Demain: A mutant of Streptomyces griseus dependent upon exogenous streptidine for streptomycin production. Bacteriol. Proc: 19, 1974

7) Wagman, G.H.; R.T. Testa \& J.A. Marquez: Antibiotic 6640. II. Fermentation, isolation and properties. J. Antibiotics 23: 555 568, 1970

8) Shier, W. T.; S. Ogawa, M. Hichens \& K. L. Rinehart, Jr.: Chemistry and biochemistry of the neomycin. XVII. Bioconversion of aminocyclitols to aminocyclitol antibiotics. J. Antibiotics 26: 551 563, 1973

9) Demain, A. L. \& E. Inamine: Biochemistry and regulation of streptomycin and mannosidostreptomycinase ( $\alpha$-D-mannosidase) formation. Bacteriol. Rev. 34: 1 19, 1970

10) WAlker, J.B. \& M.S. WALKeR: Streptomycin biosynthesis. Transamination reactions involving inosamines and inosadiamines. Biochem. 8: 763 770, 1969 